\title{
LA FUNCIÓN DEL ARTE EN LA FENOMENOLOGÍA
}

\author{
DE LA DONACIÓN DE J.-L. MARION ${ }^{1}$
}

\author{
THE ROLE OF ART IN J.-L. MARION'S \\ PHENOMENOLOGY OF GIVENNESS
}

\author{
Jorge Luis Roggero \\ Universidad de Buenos Aires \\ Instituto de Filosofía Alejandro Korn \\ jorgeluisroggero@gmail.com
}

\begin{abstract}
Resumen: Este artículo se propone indagar en la función del arte en la fenomenología de la donación de Jean-Luc Marion y su relación con la hermenéutica. La pintura desempeña un papel fundamental pues constituye el "modelo" que permite entender el carácter radicalmente pasivo de la administración hermenéutica de la distancia entre lo que se da y lo que se muestra.
\end{abstract}

Palabras clave: Marion, arte, hermenéutica.

\begin{abstract}
This article aims to investigate the role of art in Jean-Luc Marion's phenomenology of givenness and its relationship with hermeneutics. Painting plays a fundamental role because it constitutes the "model" that facilitates understanding the radically passive character of the hermeneutic management of the gap between what gives itself and what shows itself.
\end{abstract}

Keywords: Marion, art, hermeneutics.

Al comienzo de La croisée du visible, Jean-Luc Marion sostiene: "La cuestión de la pintura no pertenece ni de entrada, ni únicamente a los pintores, menos aún sólo a los estetas. Ella pertenece a la visibilidad misma y, por lo tanto, a todos, a la sensación común". ${ }^{2}$ La "cuestión de la pintura" es una cuestión deci-

\footnotetext{
${ }^{1}$ Este artículo recoge los resultados de mi estancia de investigación en el Institut Catholique de París: bajo la dirección de E. Falque, en enero y febrero de 2015. Una versión resumida de este texto fue presentada como ponencia en la Conference "Breached Horizons: The Work of Jean-Luc Marion", Londres, Canadá, 27 al 29 de marzo de 2015.

2 Marion, J.-L. (1991), La croisée du visible, Paris: Éditions de la Différence, 7. Todas las traducciones castellanas de las citas de textos originales en francés, alemán o inglés son de mi autoría. En los casos en que existen traducciones al español disponibles, han sido consultadas.
} 
siva que excede el marco de análisis de la estética o la teoría del arte pues en ella se decide la visibilidad misma, la manifestación del fenómeno. En su reciente libro sobre Courbet, Marion vuelve sobre esta idea y aclara en qué consiste la importancia fundamental del arte: "Concebir este privilegio del fenómeno -hacer aparecer la cosa en sí, el en-sí de la cosa- constituye la única tarea de la fenomenología. Pero la pintura, más que cualquier otra actividad del espíritu, tiene a su cargo llevar a cabo, poner en obra este prodigio". ${ }^{3}$

La pintura es la encargada de "hacer aparecer", de "hacer ver", de mostrar lo que se da. En términos de La croisée du visible, el pintor es el "guardián de los límites del aparecer", ${ }^{4}$ pues es quien "filtra el acceso de lo invisto (l'invu) a lo visible". ${ }^{5}$ Esta es la función del pintor: mostrar lo que sin él permanecería invisto. ${ }^{6}$

En febrero de 2013, Marion dicta la conferencia "Donation et herméneutique" en el marco de la Père Marquette Lectures in Theology. ${ }^{7}$ Allí aborda una cuestión largamente esperada por los comentaristas de su obra: finalmente, explicita cuál es la función de la hermenéutica en la fenomenología de la donación. "La hermenéutica administra la distancia entre lo que se da y lo que se muestra, interpretando la llamada (o intuición) por medio de la respuesta (concepto o significación)."

\footnotetext{
${ }^{3}$ Marion, J.-L. (2014), Courbet ou la peinture à l'œil, París: Flammarion, 10.

${ }^{4}$ Marion, J.-L., La croisée du visible, 52.

${ }^{5}$ Idem.

6 Marion distingue el concepto de "invisto" (invu) del concepto de "invisible" (invisible). "Lo invisto depende ciertamente de lo invisible, pero no se confunde con él, puesto que puede transgredirlo deviniendo precisamente visible; mientras que lo invisible permanece para siempre invisible -irreductible recalcitrante a la puesta en escena, a la aparición, a la entrada en lo visible-, lo invisto, invisible solo provisionalmente, ejerce toda su exigencia de visibilidad para, a veces por la fuerza, hacer irrupción. Lo invisto no deja de surgir incesantemente en lo visible." Ibid., 51.

7 Marion, J.-L. (2013), Givenness and Hermeneutics, Milwaukee, Marquette University Press. Esta conferencia es una ampliación del texto: "Quelques précisions sur la réduction, le donné, I'herméneutique et la donation", leído en marzo de 2011 con ocasión del Coloquio "Nouvelles phénoménologies en France", publicado posteriormente en Sommer, Ch. (ed.) (2014), Nouvelles phénoménologies en France. Actes des journées d'étude autour de Hans-Dieter Gondek et László Tengelyi Neue Phänomenologie in Frankreich, París: Hermann, 215-234.

${ }^{8}$ Desde la publicación de Réduction et donation, diversos autores han señalado la necesidad de esclarecer este punto. Cfr. entre otros, Greisch, J., "L'herméneutique dans la 'phénoménologie comme telle': trois questions à propos de 'Réduction et donation'", Revue de métaphysique et de morale, 96 (1991), 43-63; Grondin, J., "La phénoménologie sans herméneutique: Jean-Luc Marion, Réduction et donation", Internationale Zeitschrift für Philosophie, 1 (1992), 146-153; MacKinlay, Sh. (2010), Interpreting Excess. Jean-Luc Marion, satured phenomena, and hermeneutics, New York, Fordham University Press; Serban, C., "La méthode phénoménologique, entre réduction et herméneutique", Les études philosophiques, 1, n० 100 (2012), 81-100.

9 Marion, J.-L., Givenness and Hermeneutics, 54.
} 
Como ya había sido aclarado en $\S 30$ de Étant donné, si bien "todo lo que se muestra debe primero darse, sucede a veces que lo que se da no logra, sin embargo, mostrarse". ${ }^{10}$ No todo lo que se da, se muestra. La mostración del fenómeno depende de la recepción del adonado (adonné) finito. Y es esta misma finitud la que demanda entender esta recepción como una hermenéutica. Pero, ¿no es esta misma tarea la que Marion había asignado al arte? ¿La labor de administrar el pasaje de lo que se da a lo que se muestra no había sido otorgada al pintor como el encargado de hacer visible lo invisto? ¿Cómo hay que entender esta superposición de funciones entre la hermenéutica y la pintura? ¿Es posible una articulación entre ambas?

Intentaré demostrar en este artículo que no hay contradicción ni superposición: la figura del artista permite entender el modo en que opera la hermenéutica marioniana, la pintura desempeña un papel fundamental pues constituye un modelo paradigmático, una vía de acceso al particular modo en que la instancia interpretativa opera en la fenomenología de la donación.

Con este objetivo, en un primer apartado me detendré en la función que Marion asigna a la hermenéutica en su fenomenología de la donación, según es introducida en "Donation et herméneutique". En un segundo apartado, indagaré en el rol que Marion le asigna a la pintura en su obra. En un tercer apartado, examinaré los rasgos de la tarea del pintor. En un cuarto apartado, me detendré en la importancia otorgada a la figura del pintor. Finalmente propondré algunas conclusiones en torno a la función de la pintura y su relación con el carácter radical de la hermenéutica marioniana.

\section{LA HERMENÉUTICA DE LA DONACIÓN}

En la conferencia de 2013, Marion postula el carácter enigmático de la donación: ella no es ni inmediata (como sense data subjetivos), ni mediata (como objetividad). Marion recuerda la pregunta del joven Heidegger: "¿Qué quiere decir 'dado', 'donación', esta palabra mágica de la fenomenología y 'piedra de tropiezo' para los otros?". ${ }^{11}$ El fenomenólogo francés ensaya una respuesta en-

\footnotetext{
${ }^{10}$ Marion, J.-L. (1997), Étant donné. Essai d'une phénoménologie de la donation, París: PUF, 425.

${ }^{11}$ Heidegger, M. (1992), Gesamtausgabe. II. Abteilung: Vorlesungen 1919-1944. Band 58. Grundprobleme der Phänomenologie, Frankfurt am Main: Vittorio Klostermann, 5. En adelante, me referiré a la
} 
fatizando la particularidad de su carácter indeterminado: "La indeterminación de lo dado quizás ofrece su única determinación correcta, la que la distingue de todo lo que la sigue: los sense data, los objetos, los conocimientos, los frutos de su acontecimiento." ${ }^{12}$

La donación es indeterminada y enigmática. Ahora bien, frente al "enigma de la donación" interviene un segundo enigma: el de la hermenéutica. Según Marion, la hermenéutica también constituye un enigma, pues -como bien indica Heidegger- ${ }^{13}$ la interpretación depende del comprender (Verstehen), y el comprender participa del carácter enigmático de lo dado (ni inmediato, ni mediato) ${ }^{14}$ El carácter de enigma nos previene de considerar a la hermenéutica como la simple solución para la determinación del sentido de lo dado. El acto interpretativo es tan complejo como la recepción de lo dado. La hermenéutica no debe ser entendida como una operación arbitraria capaz de modificar objetos o sense data a voluntad. "La hermenéutica no da un sentido a lo dado, fijándolo y decidiéndolo, sino que, cada vez, da su sentido, es decir, da lo que hace aparecer a esto dado como él mismo, como un fenómeno que se muestra en sí y para sí."15

Se trata entonces, más que de dar sentido, de darle su sentido, de recibir el sentido dado por el fenómeno mismo. Es más, el hermeneuta sólo puede interpretar lo dado en un fenómeno si él mismo se deja interpretar por lo dado a fenomenalizar. La recepción y la interpretación se coimplican. Marion destaca que Hans-Georg Gadamer acierta al señalar la imbricación entre lo dado y el hermeneuta en una estructura de interpretación recíproca: la estructura pregunta-respuesta o llamada-respuesta. ${ }^{16}$ Esta estructura permite postular una primera tesis: "la hermenéutica debe comprenderse de acuerdo con lo dado, bajo las figuras de la llamada y la respuesta. Lejos de que la hermenéutica sobrepase la donación o la sustituya, ella se despliega en la donación casi

edición de la obra completa de Martin Heidegger con las siglas GA (Gesamtausgabe), agregando el número de tomo en cada caso.

12 Marion, J.-L., Givenness and Hermeneutics, 38.

${ }^{13}$ Cfr. GA 2, 190-213.

${ }^{14}$ Cfr. Marion, J.-L., Givenness and Hermeneutics, 38.

${ }^{15}$ Ibid., 40.

${ }^{16}$ Cfr. Gadamer, H.-G. (1990), Gesammelte Werke. Band 1. Hermeneutik I. Wahrheit und Methode. Grundzüge einer philosophischen Hermeneutik, Tübingen: J. C. B. Mohr, 375-384.

Investigaciones Fenomenológicas, n. 13, 2016. 
como un caso particular de la relación originaria entre lo que se da y lo que se muestra." 17

Por este motivo, Marion sostiene que puede hablarse de un estatuto fenomenológico de la hermenéutica, pero nunca de un estatuto hermenéutico de la fenomenología. Este punto es decisivo pues es fundamental entender que la interpretación depende de la comprensión, que comprender algo no consiste nunca en decidir de manera arbitraria sobre el sentido de un objeto. El error de entender la hermenéutica como mera enunciación arbitraria implica un ceder frente a la apariencia de la anterioridad incondicionada del "en tanto que" apofántico (apophantischer "Als"). ${ }^{18}$ Marion formula una segunda tesis:

si la hermenéutica se origina en la comprensión, si la comprensión siempre quiere decir pre-comprensión y, por lo tanto, apertura del Dasein a su posibilidad, pero si, por otra parte, la posibilidad abre al juego de la llamada y la respuesta, entonces se entrevé cómo la hermenéutica puede articularse sobre la cuestión de la donación. No es sino cuando la recepción y la identificación de lo dado implican que esto dado permanezca siempre pendiente de interpretación en tanto que fenómeno, que la instancia hermenéutica fija el lugar de lo dado, porque ella misma [la instancia hermenéutica] se fija allí. ${ }^{19}$

La necesaria participación del adonado introduce una finitud que demanda una hermenéutica. Lo dado sólo se muestra en la respuesta del adonado y el adonado es capaz de ver lo dado en tanto él mismo se recibe como adonado de esto dado. La infinitud de la donación debe pasar por el tamiz de la finitud del adonado para alcanzar su manifestación. Existe una distancia entre lo que se da y lo que se muestra que no es más que el resultado de la finitud misma del adonado.

Marion concluye la conferencia afirmando que esta tarea gestora de la hermenéutica ya se encuentra operando en sus obras, de cuatro maneras diversas.

1) En primer lugar, la hermenéutica tiene a su cargo interpretar no sólo el contenido de la llamada, sino su realidad misma. Al interpretar si la llamada dada se muestra o no, la hermenéutica decide si hay algo dado o no.

\footnotetext{
17 Marion, J.-L., Givenness and Hermeneutics, 44-46.

${ }^{18} \mathrm{Cfr} . \mathrm{GA} 2,210$.

19 Marion, J.-L., Givenness and Hermeneutics, 50.
} 
2) En segundo lugar, la hermenéutica es necesaria para recorrer la distancia entre el "fenómeno saturado" (phénomène saturé) y sus significaciones. En De surcroît, Marion invoca una "hermenéutica infinita" ante las infinitas interpretaciones posibles que demanda la intuición saturante del fenómeno del rostro. "El rostro del otro requiere una hermenéutica infinita, equivalente al 'progreso al infinito' de la moralidad según Kant. Pues todo rostro exige la inmortalidad." 20

3) En tercer lugar, la hermenéutica actúa para distinguir los diversos grados de intuición. La "respuesta hermenéutica" del adonado, presentada en Le visible et le révélé, devela la "banalidad" de la saturación. "El adonado no es pasivo en absoluto, puesto que por su respuesta (hermenéutica) a la llamada (intuitiva), él y sólo él, permite a lo que se da devenir, en parte solamente pero realmente, lo que se muestra." 21

4) En cuarto lugar, radicalizando la banalidad de la saturación, la hermenéutica hace posible la distinción última entre objetos y acontecimientos introducida en Certitudes négatives, haciéndola depender de "variaciones hermenéuticas". "La distinción de los modos de la fenomenicidad (para nosotros entre objeto y acontecimiento) puede articularse sobre variaciones hermenéuticas". ${ }^{22}$

Con esta enumeración de "cuatro momentos hermenéuticos de la donación", Marion establece de manera explícita la función de la hermenéutica en su fenomenología de la donación. Ella cumple un papel decisivo: ser la encargada de administrar, de gestionar el pasaje de lo que se da a lo que se muestra. Ahora bien, ¿cómo funciona esta hermenéutica? ¿Cómo opera esta interpretación que es también un dejarse interpretar? ¿Cómo debe entenderse esta tarea "creativa" que se limita a "descubrir" lo ya dado?

\section{LA FUNCIÓN DE LA PINTURA}

La tarea de administrar la distancia entre lo que se da y lo que se muestra no es un tópico nuevo en la obra de Marion, introducido con la mención a la hermenéutica en la conferencia de 2013. Hacer visible lo invisto, fenomenalizar

\footnotetext{
${ }^{20}$ Marion, J.-L. (2001), De surcroît. Études sur les phénomènes saturés, París: PUF, 152.

${ }^{21}$ Marion, J.-L. (2005), Le visible et le révélé, París: Cerf, 181.

22 Marion, J.-L. (2010), Certitudes négatives, París: Grasset, 307.
} 
lo dado es la función que Marion asigna a la pintura desde la publicación de los estudios que componen La croisée du visible en 1991.

En "Ce que cela donne", Marion se pregunta para qué necesitamos de los pintores, cuál es su función específica. Está claro que "para ver, no tenemos ninguna necesidad de los pintores", ${ }^{23}$ vemos "el espectáculo visible" de la cotidianeidad sin necesidad de recurrir a la pintura: "sabemos lo que vemos y lo que debemos ver"; ${ }^{24}$ evitamos cualquier tipo de sorpresa: procuramos que lo que vemos sea lo que tenemos que ver; nos aseguramos tan sólo de que lo que vemos coincida con lo que debemos ver. Pero entonces, ¿qué puede aportar la pintura respecto de esta visibilidad?

$Y$ en este punto, la respuesta de Marion es terminante: la pintura pone en jaque la apacible "coincidencia" entre lo que creemos que tenemos que ver y lo que vemos. La pintura nos muestra otra cosa: "El cuadro -el verdaderoexpone un fenómeno absolutamente original, descubierto por primera vez, sin precedente ni genealogía, surgido con una violencia tal que hace explotar los límites del repertorio de lo visible hasta el momento."25

En De surcroît, Marion indaga en las características fenomenológicas del cuadro a partir de una frase de Blaise Pascal: "iQué vanidad la de la pintura que atrae la admiración por su semejanza con cosas cuyos originales no son admirados!" ${ }^{26}$ ¿Cómo deben interpretarse estas palabras? Marion se detiene en la "semejanza" (ressemblance) entendida, no como una relación, sino como uno de los términos de la relación con el original. La "semejanza" de la pintura tiene la particularidad de confiscar el resplandor del original, de devenir ella misma el original. Éste es el poder de la pintura: 1) desplazar la admiración ${ }^{27}$ del mundo físico al arte: "la admiración se concentra pues en la semejanza, justamente porque ella ya no se asemeja a nada", ${ }^{28}$ y 2) confiscar la fenomenicidad: ${ }^{29}$ "La pintura no redobló o adecuó la fenomenicidad, la dominó (en

\footnotetext{
${ }^{23}$ Marion, J.-L., La croisée du visible, 49.

${ }^{24}$ Idem

25 Ibid., 50.

${ }^{26}$ Pascal, B. (1963), Pensées, París: Seuil, 508.

27 Marion define la "admiración" (admiration) como "el más potente ejercicio posible de la mirada." El ejercicio que "fija [la mirada] de forma estable, casi fascinada, sobre lo que encuentra, o más bien, le adviene, en lugar de vagar a la manera de la simple vista, que erra de un visible a otro sin retrasarse". Marion, J.-L. (2001), De surcroît. Études sur les phénomènes saturés, París: PUF, 2001, 71.

${ }^{28}$ Ibid., 70.

29 Sigo la sugerencia de Javier Bassas Vila respecto de la traducción de phénoménalité por "fenomenicidad". Bassas Vila, J. "Glosario" en Marion, J.-L. (2008), Siendo dado. Ensayo para una fenomenología de la donación, Madrid: Síntesis, 506.
} 
detrimento de la naturaleza, del original), la produjo (instituyendo el privilegio de la semejanza) y finalmente la consagró desplazando el centro de gravedad de la semblanza pura." 30

La pintura se apropia de la fenomenicidad, pero, ¿cómo es esto posible?

Marion responde que esto se logra por medio de dos características del cuadro. 1) En primer lugar, el marco, el enmarcamiento propio del cuadro permite hacer un recorte en lo visible. 2) En segundo lugar, precisamente a partir de este encuadramiento, la mirada queda presa de su ídolo, ${ }^{31}$ queda presa de una visibilidad que la excede, la satura y acapara toda su admiración. Esto es posible pues, a diferencia de lo que aparece en el mundo, que se compone siempre de presentación y apresentación, el cuadro

reduce el objeto a presentable en sí, excluye de él lo apresentable; resumiendo, [el cuadro] desmonta el objeto para reducirlo a lo visible en sí, a lo visible puro y sin resto; en el cuadro, no queda más que lo visible enteramente presentado, sin ya prometer nada más para ver que lo que ya se ofrece. Ese visible reducido, presentado en estado puro, sin ningún resto de apresentación, logra una intensidad tal que frecuentemente satura la capacidad de mi mirada, incluso la excede. ${ }^{32}$

Este visible reducido tiene la intensidad de un nuevo visible, de un invisto que asciende por primera vez a la visibilidad. La pintura "produce [...] un visible que antes no había sido visto por nadie". ${ }^{33}$

En su libro sobre Courbet, Marion reitera la idea de una reducción del cuadro a lo visible, ${ }^{34}$ que permite lograr un acceso a "lo que se da realmente", a las "cosas reales y existentes". ${ }^{35}$ Pero para poner en práctica esta tarea, la pintura de Courbet debe "destruir lo que obstaculiza el ver 'de inmediato', [debe] atravesar el velo de transparencia oscura que disimula las cosas tal como son". ${ }^{36}$

\footnotetext{
${ }^{30}$ Marion, J.-L., De surcroît, 71.

${ }^{31}$ En Étant donné, Marion postula el estatuto de fenómeno saturado del cuadro. Marion, J.-L., Étant donné, 320. En De surcroît, Marion examina la especificidad de los fenómenos saturados presentados en Étant donné. El tercer estudio se aboca al análisis del ídolo (idole) en la figura del cuadro. "El ídolo se define aquí como el primer término indiscutiblemente visible porque su esplendor detiene por vez primera la intencionalidad; y ese primer visible la colma, la detiene e incluso la bloquea hasta el punto de volverla contra ella misma, a la manera de un obstáculo -o un espejo- invisible". Marion, J.-L., De surcroît, 71.

32 Marion, J.-L., De surcroît, 76-77.

${ }^{33}$ Ibid., 82.

34 Marion, J.-L., Courbet, 163.

35 "La pintura es un arte esencialmente concreto y no puede consistir más que en la representación de las cosas reales y existentes". Courbet, G., "Aux jeunes artistes de Paris" en Ten-Doesschate Chu, P. (éd.) (1996), Correspondance de Courbet, París: Flammarion, 183.

${ }^{36}$ Marion, J.-L., Courbet, 46.
}

| Investigaciones Fenomenológicas, n. 13, 2016. 
Courbet pone en práctica una suerte de heideggeriana Destruktion hermenéuti$\mathrm{ca}^{37}$ que le permite ejecutar la reducción. Cotidianamente no "vemos" las cosas. Vemos objetos, pero no cosas: "...los objetos son concebidos "desde la idea" [à l'idée], por una mirada que los domina totalmente y los produce a voluntad, mientras que la cosa se impone desde ella misma al pintor tanto como al espectador." 38

Marion sostiene que el arte tiene la función de "cumplir" (accomplir) la cosa. Este "cumplir" no equivale a imponer una forma, sino a "cumplir la forma que le falta a lo invisto para que él ascienda desde sí mismo a lo visible. Esto implica que una mirada distinga esta forma aún escondida en lo invisto de la cosa y la libere". ${ }^{39}$ Es necesario pues liberar a la cosa, quitarle el "velo" "objetivo" que la disimula para que ésta advenga en tanto tal.

\section{LOS RASGOS DE LA FUNCIÓN DEL PINTOR}

Marion sostiene que el pintor es el indicado para "filtrar" el acceso de lo invisto a lo visible pues no se trata simplemente de la visión de lo visible, sino de la adivinación de lo invisto (divination de l'invu). Como un ciego (aveugle) o un vidente (voyant), "el pintor ve más que lo visible". ${ }^{40} \mathrm{Y}$ puede "ver más" porque asume el riesgo de "descender a la frontera indecisa de lo visible y lo invisto", de internarse en la oscuridad hasta perderse a sí mismo. El pintor va al encuentro de lo invisto, al encuentro de aquello que no tiene modelo ni precedente. $Y$ esto implica exponerse al peligro más extremo. Marion compara al pintor con la figura de Cristo:"El pintor debe perderse para salvar (y salvarse). Como Cristo, él sólo acoge y salva porque él se da primero, sin saber jamás por adelantado si él se pierde o se salva a sí mismo." ${ }^{41}$

La "mirada ciega" (regard aveugle) del pintor se aparta de toda previsión, de toda operación que provenga de su sí mismo, para entregarse a lo imprevisible por definición: lo invisto. Esta entrega implica un "perderse" a partir de una pasividad radical.

\footnotetext{
37 "iHermenéutica es destrucción!" GA 63, 105.

38 Marion, J.-L. Marion, Courbet, 128.

${ }^{39}$ Ibid., 132.

${ }^{40}$ Marion, J.-L., La croisée du visible, 52.

${ }^{41}$ Ibid., 54.
} 
Ahora bien, paradójicamente, esta pasividad no implica que su tarea consista en un mero "reproducir" (reproduire), sino que, por el contrario, su función debe ser entendida como un "producir" (produire). El pintor "produce" y no simplemente "reproduce" algo ya visible, porque introduce un invisto en el campo de lo visible: "Producir el cuadro no equivale pues jamás a hacer efectiva una visibilidad prevista, preconcebida y ya vista antes incluso de que surja sensiblemente a la luz. Bajo esta acepción, toda producción se rebajaría al rango de una reproducción, en el que lo efectivamente visible se limita a imitar lo visible en potencia." ${ }^{42}$

Pero, ¿cuál es el estatuto de esta "producción"? ¿Puede hablarse de "creación"? Marion afirma que "el verdadero pintor participa del simple misterio de la única Creación, por el hecho de que no reproduce nada, sino que produce". ${ }^{43}$ Sin embargo, si bien puede sostenerse que hay una actividad "creadora" involucrada, no puede asignarse al pintor el rango de "creador". "El verdadero pintor no sabe lo que ha pintado". ${ }^{44} \mathrm{Y}$ esto es así, porque no es él quien crea, desde su "querer", la pintura.

El pintor, al contrario, no se permite ni hacer ver lo que quiere ni dejar ver lo que no quiere, puesto que intenta no poder ya hacer (ni hacer ver) lo que aún podría querer o dominar. Él intenta dejar surgir mucho más que lo previsto, que lo visto, que lo querido. 0 , más bien, él sólo hace lo que quiere -dejar aparecer (desaparecer pues inmediatamente como tal) un invisto en lo visible- si renuncia a hacer lo que podría, sin embargo, perfectamente efectuar, un objeto previsto. ${ }^{45}$

El "verdadero" pintor crea de un modo particular, crea deshaciéndose de su voluntad, deshaciéndose de sí mismo. Su "creación" tiene un carácter paradójico pues no es activa, sino pasiva: "El pintor verdaderamente creador no se caracteriza tanto por una inventiva plástica que impone su elección, sino por una pasividad receptiva que, entre mil trazos igualmente posibles, sabe elegir el que se impone por su necesidad propia." ${ }^{46}$

\footnotetext{
42 Ibid., 54-55.

${ }^{43}$ Ibid., 55-56.

44 Ibid., 60.

${ }^{45}$ Ibid., 61.

${ }^{46}$ Ibid., 66-67.
}

Investigaciones Fenomenológicas, n. 13, 2016. 
Sólo a partir de esta "pasividad receptiva" el pintor puede asegurarse que la creación no sea arbitraria. "El pintor registra, no inventa nada". 47 El pintor no imita una realidad existente pero tampoco la "inventa", sino que descubre la huella (trace) que lo invisto impone. "En el cuadro, y a la medida de la sensibilidad fiel y resistente del pintor, se consignan los estigmas [stigmates] de lo invisto a partir de ellos mismos". ${ }^{48}$ Marion llama a estos estigmas "ectipos" (ectypes), marcas internas que ascienden desde el "fondo del cuadro". El creador del cuadro es el propio cuadro que se organiza y surge a la visibilidad en sus ectipos, según el registro del pintor. ${ }^{49}$ Marion resume la labor de este pintor: "El pintor no tiene que producir formas. Él debe registrar, mediante una segunda y difícil pasividad (la del arte de no hacer nada), el surgimiento de los ectipos a partir de su fondo, el ascenso de lo invisto hacia el aparecer. ${ }^{\prime 50}$ El registro pasivo, el seguimiento de las huellas de lo invisto como el tanteo de un ciego con su bastón, ${ }^{51}$ caracterizan la labor "creativa" del pintor, pues "pintar quiere decir: esperar una donación". ${ }^{52}$

En su libro de 2014, Marion presenta la figura de Courbet como una suerte de pintor paradigmático. "Así como ciertos músicos tienen el oído absoluto, ciertos pintores tendría el ojo absoluto [I'œil absolu]: ellos verían mejor, con más nitidez las formas, con más exactitud la intensidad de los colores, más lúcidamente las composiciones, etc. ${ }^{53}$ Según Marion, Courbet no sólo tenía un ojo absoluto, sino que "era un ojo": "ser (no solamente tener) un ojo no significa ver primero, y después pintar (o no pintar), pintar viendo, pintar después de haber visto; sino que ser un ojo significa ver pintando [voir en peignant], ver al mismo tiempo, en el mismo gesto y con la misma energía que se pinta; dicho de otro modo, ver en tanto que pintando." ${ }^{\prime 4}$

Courbet "ve pintando". El gesto de pintar precede toda mirada identificadora de la cosa. Courbet pinta lo que ve antes aún de saber de qué objeto se tra-

\footnotetext{
47 Ibid., 67.

${ }^{48}$ Ibid., 68.

${ }^{49}$ Cfr. Ibid., 69.

${ }^{50}$ Ibid., 74.

${ }^{51}$ Cfr. Ibid., 67.

52 Ibid., 80.

53 Marion, J.-L., Courbet, 25.

54 Ibid., 25.
} 
ta. Courbet pinta "desde el ojo" (à l'œil). ${ }^{55}$ "Ver pintando" implica pintar "desde el ojo" y no "desde la idea". La pintura "desde la idea" - representada paradigmáticamente por Ingres en tiempos de Courbet- no parte del surgimiento del fenómeno desde sí mismo y que es recibido como tal por el "ojo", sino de un concepción previa, de una idea que pretende pintar antes de ver, que pretende imponer una forma, por medio del dibujo, o constituir un objeto según la composición en lugar de entregarse al advenimiento a la visibilidad de lo invisto. No se trata pues, para el pintor, de duplicar con "arte", con "técnica", la aparición de la cosa, sino de "dejar surgir lo visible y cumplirse con él en una única energía, haciendo accesible para el espectador el ver lo que no había previsto". ${ }^{56} \mathrm{El}$ pintor debe "ver pintando" sin modelo pre-visto, sin pre-ver. Se trata de "pintar para ver" (peindre pour voir). En este sentido, Courbet (como todos los pintores dignos de ese nombre) se inscribe entre los practicantes del fenómeno saturado, de la aparición de un fenómeno donde el exceso y la prioridad de la intuición no pueden jamás dejarse reglar por uno o varios conceptos, significaciones o concepciones que los precedan. ${ }^{57}$ Contraponiéndose a la lógica cartesiana, Marion podría afirmar: "pinto, luego no existo". Pintar exige neutralizar el ego, suspender la conciencia objetivante, abandonar la función dominadora y constitutiva del sujeto para someterse a la cosa. "Si yo no soy más que en tanto que pienso, desde el momento en que no pinto más que a condición de no pensar nada (ni teoría, ni interpretación, ni voluntad de intervención), casi de no pensar, no pinto correctamente más que en tanto que no soy. ${ }^{158}$ Courbet, el pintor por excelencia, pone en práctica una suerte de "ascesis activa" (ascèse active $)^{59}$ que le permite ver y hacer ver la cosa misma, dejándose afectar por ella.

Hasta aquí, la función asignada a la pintura y el modo en el que el pintor desempeña su labor parecen constituir un modelo adecuado para pensar la tara hermenéutica. Sin embargo, Marion dice algo más. Marion inviste al pintor con

\footnotetext{
${ }^{55}$ Esta expresión es de difícil traducción, opto por la preposición "desde" para indicar la procedencia, en contraposición a la pintura hecha "desde la idea" (à l'idée), pero es importante tener en cuenta el doble sentido del sintagma que Marion pretende conservar. Se trata de pensar un fenómeno que surge à l'œil, "en el doble sentido de lo que se impone, lo que captura la atención (¿lo que seduce? [fait de l'œil?]) y [lo que] se da desde sí mismo, por su pura y propia gracia (gratuitamente [à l'œil], gratis). Ibid., 27.

${ }^{56}$ Ibid., 28-29.

57 Ibid., 29.

${ }^{58}$ Ibid., 191.

${ }^{59}$ Cfr. Ibid., 165.
}

Investigaciones Fenomenológicas, n. 13, 2016. 
un poder especial. Pareciera que su función no es meramente la de servir de modelo, sino la de ser el único adonado capaz de realizar efectivamente la reducción de lo visible:

El cuadro no ofrece un ejemplo, interesante, pero eventualmente facultativo, del método fenomenológico de la reducción. El cuadro lo realiza radicalmente según la cualidad (la intensidad o la magnitud intensiva) del aparecer. Reduciendo lo visible a su quintaesencia atómica, conteniendo en su marco la energía demencial de lo visible, el cuadro reduce lo que se da a lo que se muestra, bajo el régimen del ído$10 .^{60}$

\section{EL PODER DEL PINTOR}

En "Ce que cela donne", Marion contrapone el pintor al filósofo. Platón advirtió con claridad el enorme poder del artista. "Platón no estaba equivocado al denunciar ese poder telúrico y apolíneo a la vez, que arrebata de antemano al filósofo su más alto oficio: decidir la presencia. Sólo el pintor da la venia apparendi, el permiso de aparecer, el derecho a la presencia. ${ }^{\prime 61}$ Es el pintor, y no el filósofo, quien tiene a su cargo velar en la frontera de lo visible. Él es el "portero que filtra el acceso de lo invisto a lo visible [...] el guardián de los límites del aparecer".62

En De surcroît, Marion vuelve sobre esta idea. El pintor es el "cazador de invistos insospechados" (chasseur d'invus insoupçonnés), en este sentido, sus obras tienen una función fundamental: "Son ellas [las obras en tanto ídolos] quienes, en cada época, reinan sobre los visibles naturales, sobre la apariencia de los objetos constituidos y nos obligan a ver todo a partir de los paradigmas que imponen su fascinación. El pintor es rey, tanto y sin duda más inmediatamente que ningún filósofo. ${ }^{\prime 63} \mathrm{El}$ "rey-pintor" tiene una función más importante que la del "rey-filósofo", pues tiene a su cargo "hacernos ver". El pintor, en tanto "practicante del fenómeno saturado", 64 es un entrenador de la vista. Ver el ídolo de la obra de arte ejercita nuestro ojo para ver lo que no vemos cotidianamente, para sospechar de la apacible "coincidencia" entre lo que creemos

\footnotetext{
${ }^{60}$ Marion, J.-L., De surcroît, 82.

${ }^{61}$ Marion, J.-L., La croisée du visible, 52.

62 Ibid., 52.

${ }^{63}$ Marion, J.-L., De surcrołt, 84.

${ }^{64}$ Ibid., 29.
} 
que tenemos que ver y lo que vemos, para ver sin pre-ver, para ver lo invisto, para esperar la donación de la cosa a partir de sí misma.

Como ya hemos mencionado en la introducción, al comienzo de Courbet..., Marion confirma este aserto de De surcroît, proponiendo una suerte de articulación entre filosofía y arte. La tarea de la filosofía es "comprender el fenómeno como aparece, o dicho de otro modo, como se da". ${ }^{65} \mathrm{Y}$ en tanto el fenómeno es aquello que se da haciendo posible la aparición de la cosa en sí, la labor de la fenomenología no es otra que la de "concebir este privilegio del fenómeno". ${ }^{66}$ Pero para realizar esta tarea es necesaria la pintura. "La pintura, más que cualquier otra actividad del espíritu, tiene a su cargo poner en obra este privilegio [del fenómeno]". ${ }^{67}$ El pintor es el encargado de poner en obra el "privilegio del fenómeno", es quien permite que el fenómeno se dé haciendo posible la aparición de la cosa en sí, es quien "administra la distancia entre lo que se da y lo que se muestra". En este sentido, el pintor es el hermeneuta por excelencia, quien hace posible el despliegue de la fenomenología de la donación en el pasaje de la donación a la mostración.

Pero, esto implica que para ver las "cosas reales y existentes", para acceder a la cosa en sí, ¿debemos devenir pintores o acaso debemos aceptar la guía del rey-pintor? ¿Solo un pintor puede ver y dar a ver?

A estos interrogantes se suma el problema de que ya no hay "pintores". Marion utiliza siempre los términos "pintura" (peinture) y "pintor" (peintre), y nunca "arte" (art) y "artista" (artiste) porque considera que el arte de nuestro tiempo, el "arte conceptual", ha perdido la capacidad de hacer ver lo invisto, pues en él lo visible es definido de antemano por el concepto y la obra no aparece en tanto tal. ${ }^{68}$

\section{ARTE Y HERMENÉUTICA}

En el $\S 1$ de Étant donné, Marion distingue entre el enfoque metafísico y el enfoque fenomenológico. El enfoque metafísico - como el enfoque de toda ciencia- asume una metodología que, para alcanzar el conocimiento, debe "fun-

\footnotetext{
65 Marion, J.-L., Courbet, 10.

${ }^{66}$ Idem.

${ }^{67}$ Idem.

${ }^{68}$ Cfr. Marion, J.-L., La croisée du visible, 55 y 64, y Marion, J.-L., Courbet, 134. Hice una lectura de esta problemática en Roggero, J. L., "Arte y concepto. La crítica de Jean-Luc Marion al arte conceptual", Eikasia. Revista de Filosofía, 66 (2015), 205-222.
}

Investigaciones Fenomenológicas, n. 13, 2016. 
damentar la apariencia", debe "reconducirla al fundamento". El enfoque metafísico se propone, entonces, "demostrar". Frente a este planteo, Marion señala que el enfoque fenomenológico tiene un propósito distinto, su objetivo no es "demostrar", sino simplemente "mostrar". "Mostrar implica dejar que la apariencia aparezca de tal manera que cumpla su plena aparición, para recibirla exactamente como se da". ${ }^{69}$ La pintura $-\mathrm{y}$ el arte en general- cumplen plenamente este propósito fenomenológico de "mostrar" en lugar de "demostrar". Como ya hemos destacado, Marion señala esta superioridad de la pintura respecto de la fenomenología y la hermenéutica: el poder del pintor es mayor al del filósofo porque sólo él puede "mostrar" sin más. ¿Pero cuál es el estatuto de este poder? Marion destaca que la "cuestión de la pintura" no pertenece sólo a los pintores sino a todos porque ésta decide la visibilidad misma. En este sentido, todos debemos devenir "pintores", todos debemos hacernos cargo de la "cuestión de la pintura" en tanto todos debemos asumir el poder de desarrollar un "ojo absoluto", el poder de hacer visible lo invisto. No se trata de aceptar la guía del "rey-pintor", tampoco - de más está decir- de realizar efectivamente la tarea de pintar un cuadro, se trata más bien de devenir "pintores" en el sentido de ser capaces de imitar su pasividad radical, su entrega al acontecer del fenómeno.

Por otra parte, la obras de arte nos "obligan a ver todo a partir de los paradigmas que imponen su fascinación" ${ }^{\prime 70}$ porque ellas constituyen fenómenos saturados. Como bien destaca Alain Bonfand, la experiencia estética es el espacio privilegiado en el que la supuesta igualdad entre intención e intuición se vuelve insostenible, ${ }^{71}$ la experiencia estética es la experiencia paradigmática del fenómeno saturado o, al menos, constituye una de las vías privilegiadas de acceso al aparecer hermenéutico del fenómeno, la vía del fenómeno saturado del ídolo. $Y$ este punto es decisivo porque la pintura como modelo paradigmático da cuenta de la imbricación entre hermenéutica y fenómeno saturado: lo que se muestra, si proviene de lo dado, si proviene de una reducción a la donación, sólo puede mostrarse como fenómeno saturado. Realizar la tarea hermenéutica implica devenir "practicantes del fenómeno saturado". Si -como indica Ma-

\footnotetext{
${ }^{69}$ Marion, J.-L., Étant donné, 13.

70 Marion, J.-L., De surcroit, 84.

${ }^{71}$ Cfr. Bonfand, A. (2009), Histoire de l'art et phénoménologie, París: Vrin, 28.
} 
rion- la hermenéutica se pone en práctica bajo cuatro modalidades, si ella conlleva 1) decidir si hay algo dado, 2) reconocer el carácter infinito de la tarea interpretativa, 3) asumir la respuesta que devela la banalidad de la saturación y 4) disponer la "variación hermenéutica", su actividad se relaciona en todos los casos, de un modo fundamental, con el fenómeno saturado. Gestar el pasaje de lo que se da a lo que se muestra implica mostrar un fenómeno saturado.

Respecto de esta tarea, la pintura, pues, cumple una función decisiva porque ofrece el modelo a seguir para la filosofía. Si la fenomenología de la donación se realiza por medio de un trabajo hermenéutico que hace posible la mostración de lo que se da, esa hermenéutica debe seguir los lineamientos de la pintura.

Así como la fenomenología de la donación ya no toma como punto de partida una instancia subjetiva constitutiva, sino que permite al fenómeno tomar la iniciativa, también la hermenéutica de la donación desplaza la intervención de algún tipo de ego trascendental a favor del advenimiento del fenómeno desde sí mismo. La conferencia de 2013 es clara al respecto: no es el sujeto hermeneuta el que decide sobre el sentido, sino que es el mismo "sí" del fenómeno quien se da su sentido. ${ }^{72}$ La figura del pintor, en tanto figura del sujeto creador por excelencia, le permite a Marion dar cuenta de que aún en este caso, tampoco es cierto que originariamente opere una actividad de constitución por parte del sujeto. El caso extremo del pintor deviene el caso paradigmático, pues Marion demuestra que el sujeto-pintor también se comporta como un adonado.

El pintor-adonado deviene el modelo de hermeneuta porque permite entender en detalle las diversas aristas que componen la tarea hermenéutica de administrar la distancia entre lo que se da y lo que se muestra. El pintor da cuenta de la compleja imbricación entre recepción e interpretación. Lo que se recibe como dado, se recibe como indeterminado y, por lo tanto, como necesitado de interpretación. El "enigma de la donación" es el enigma de la indeterminación de lo dado que demanda la intervención de una hermenéutica. Ahora bien, esta necesidad de interpretación no implica la introducción de la arbitrariedad. La tarea del pintor da cuenta de una hermenéutica que se impone por la necesidad de la cosa misma. Pero, ¿cómo opera esta interpretación "necesaria"?

${ }^{72}$ Cfr. Marion, J.-L., Givenness and Hermeneutics, 40.

Investigaciones Fenomenológicas, n. 13, 2016. 
El pintor "filtra" el acceso de lo invisto a lo visible porque no hace nada, porque se entrega al fenómeno. La hermenéutica de la donación marioniana no parte de la voluntad creadora del sujeto intérprete, sino del "sí" del fenómeno. El pintor crea sin querer, interpreta dejando que el fenómeno se dé a sí mismo su sentido. El pintor-hermeneuta no inventa, no impone sentidos arbitrarios, no impone conceptos a priori (no pinta "desde la idea"), sino que registra el acontecer del fenómeno y su sentido. El pintor pone en obra esta actividad/pasividad que le permite hacer de la recepción una interpretación mejor que cualquier otra pues se trata del adonado dotado con mayor "pasividad receptiva", es quien tiene el ojo mejor entrenado. El pintor transforma lo invisto en visible porque ve mejor, más intensamente, desde un ojo que se deja afectar por el advenimiento de lo imprevisto.

Ahora bien, esta pasividad radical es también una acción. El pintor es un activista de la "pasividad receptiva" pues es esta pasividad la que le permite "destruir lo que obstaculiza el ver", atravesar el velo objetivo que disimula las cosas tal como son. Su acción radical consiste en no hacer, en dejar hacer al fenómeno. Analizando la noción de "variación hermenéutica" y la clasificación de los fenómenos en objetos y acontecimientos, introducidas en Certitudes négatives, Claudia Serban se pregunta: "¿Podemos aceptar que la mirada decide el acontecimiento y conservar el compromiso de Étant donné a favor del "sí" del fenómeno, compromiso que estatuye que la "iniciativa pertenece en principio al fenómeno y no a la mirada"? ${ }^{73}$

La respuesta está dada por la "ascesis activa" del pintor: no es la decisión del hermeneuta, sino la entrega absoluta a la iniciativa del "sí" del fenómeno la que da lugar a la "variación hermenéutica" que permite recibir la cosa como acontecimiento, en su fenomenicidad saturada. No es la mirada la que decide, sino el fenómeno mismo al que el hermeneuta "decide" entregarse.

Esta entrega, que conlleva una "neutralización del ego", es la permite operar a la hermenéutica marioniana. La interpretación pasivo/receptiva, la "ascesis activa", sólo se logra si el sujeto no hace nada. "No hacer nada" implica asumir el riesgo de perderse a sí mismo, de entregarse a lo que ya no puede

\footnotetext{
${ }^{73}$ Serban, C., "La méthode phénoménologique", 92.
} 
prever ni controlar, de dejarse interpretar deviniendo testigo y no ya artífice de la "creación".

La pintura cumple una función fundamental pues constituye el "modelo" que permite entender el carácter radicalmente pasivo de la administración hermenéutica de la "distancia entre lo que se da y lo que se muestra", de la gestión del pasaje de lo invisto dado a lo visible. Ahora bien, de más está decir que nuestra hipótesis es sólo una interpretación posible que pretende explicar una cuestión que no ha sido explicitada, pues Marion no ha aclarado en qué consiste la relación entre el arte y la hermenéutica en su obra. La pregunta respecto del estatuto del pintor y de la pintura en el proyecto filosófico marioniano permanece pues a la espera del pronunciamiento del propio autor, y se vuelve más urgente aún si tenemos en cuenta que vivimos en los tiempos del "arte conceptual" que tan severamente ha sido condenado por Marion. ¿Cómo seguir el modelo de la pintura cuando ya no hay pintores? ¿Quién podrá establecer los paradigmas de la visibilidad para nuestro tiempo?

\section{BiBLIOGRAFIA}

Bonfand, A. (2009), Histoire de l'art et phénoménologie, Paris: Vrin.

Courbet, G. (1996), "Aux jeunes artistes de Paris" en Ten-Doesschate Chu, P. (éd.), Correspondance de Courbet, Paris: Flammarion.

GadAmer, H.-G. (1990), Gesammelte Werke. Band 1. Hermeneutik I. Wahrheit und Methode. Grundzüge einer philosophischen Hermeneutik, Tübingen: J. C. B. Mohr. Traducción al español: Gadamer, H.-G. (1977), Verdad y método. Fundamentos de una hermenéutica filosófica, trad. A. Agud Aparicio y R. de Agapito, Salamanca: Sígueme.

GREISCH, J. (1991), "L'herméneutique dans la 'phénoménologie comme telle': trois questions à propos de 'Réduction et donation'", Revue de métaphysique et de morale, 96, 43-63.

GRondiN, J. (1992), "La phénoménologie sans herméneutique: Jean-Luc Marion, Réduction et donation", Internationale Zeitschrift für Philosophie, 1, 146153.

Heidegger, M. (1992), Gesamtausgabe. II. Abteilung: Vorlesungen 1919-1944.

Band 58. Grundprobleme der Phänomenologie, Frankfurt am Main: Vittorio 
Klostermann (GA 58). Traducción al español: Heidegger, M. (2014), Problemas fundamentales de la fenomenología (1919/1920), trad. F. de Lara, Madrid: Alianza.

- (1988), Gesamtausgabe. II. Abteilung: Vorlesungen 1919-1944. Band 63. Ontologie. Hermeneutik der Faktizität, Frankfurt am Main: Vittorio Klostermann (GA 63). Traducción al español: HeIdegGer, M. (1998), Ontología. Hermenéutica de la facticidad, trad. J. Aspiunza, Madrid: Alianza.

- (1977), Gesamtausgabe. I. Abteilung: Veröffentlichte Schriften 19101976. Band 2. Sein und Zeit, Frankfurt am Main, Vittorio Klostermann (GA 2). Traducciones al español: Heidegger, M. (1951), El ser y el tiempo, trad. J. Gaos, México, FCE, y Heidegger, M. (2003), Ser y tiempo, trad. Jorge Eduardo Rivera Cruchaga, Madrid: Trotta.

MACKINLAY, Sh. (2010), Interpreting Excess. Jean-Luc Marion, satured phenomena, and hermeneutics, New York: Fordham University Press.

MARION, J.-L. (1991), La croisée du visible, Paris, Éditions de la Différence. Traducción al español: MARION, J.-L. (2006), El cruce de lo visible, trad. J. Bassas Vila y J. Masó, Castellón: Ellago Ediciones.

- (1997), Étant donné. Essai d'une phénoménologie de la donation, Paris, PUF. Traducción al español: MARION, J.-L. (2008), Siendo dado. Ensayo para una fenomenología de la donación, trad. J. Bassas Vila, Madrid: Editorial Síntesis.

- (2001), De surcroît. Études sur les phénomènes saturés, Paris: PUF.

- (2005), Le visible et le révélé, Paris: Cerf.

- (2010), Certitudes négatives, Paris: Grasset.

- (2013), Givenness and Hermeneutics, Milwaukee: Marquette University Press.

- (2014), Courbet ou la peinture à l'œil, Paris: Flammarion.

PASCAL, B. (1963), Pensées, Paris, Seuil. Traducción al español consultada: Pascal, B. (1981), Pensamientos, trad. J. Llansó, Madrid: Alianza.

Roggero, J. L., "Arte y concepto. La crítica de Jean-Luc Marion al arte conceptual", Eikasia. Revista de Filosofía, 66 (2015), 205-222.

SERBAN, C. (2012), "La méthode phénoménologique, entre réduction et herméneutique", Les études philosophiques, 1, n 100, 81-100. 
Sommer, Ch. (éd.) (2014), Nouvelles phénoménologies en France. Actes des journées d'étude autour de Hans-Dieter Gondek et László Tengelyi Neue Phänomenologie in Frankreich, Paris: Hermann.

| Investigaciones Fenomenológicas, n. 13, 2016. 